\title{
On the stability of a mixed type quadratic-additive functional equation
}

\author{
Young-Su Lee*, Junyeop Na and Heejong Woo
}

${ }^{\text {*Correspondence: }}$ masuri@kaist.ac.kr

Hana Academy Seoul, Seoul,

122-200, Republic of Korea

\section{Abstract}

In this paper we establish the general solutions of the following mixed type quadratic-additive functional equation:

$$
9 f\left(\frac{x+y+z}{3}\right)+4\left[f\left(\frac{x-y}{2}\right)+f\left(\frac{y-z}{2}\right)+f\left(\frac{z-x}{2}\right)\right]=3[f(x)+f(y)+f(z)]
$$

in the class of functions between real vector spaces. Moreover, we prove the generalized Hyers-Ulam-Rassias stability of this equation in Banach spaces.

MSC: 39B82; 39B52

Keywords: quadratic functional equation; additive functional equation; mixed type functional equation; stability

\section{Introduction}

The stability problems of functional equations go back to 1940 when Ulam [1] proposed the following question:

Let $f$ be a mapping from a group $G_{1}$ to a metric group $G_{2}$ with the metric $d(\cdot, \cdot)$ such that

$$
d(f(x y), f(x) f(y)) \leq \epsilon .
$$

Then does there exist a group homomorphism $L: G_{1} \rightarrow G_{2}$ and $\delta_{\epsilon}>0$ such that

$$
d(f(x), L(x)) \leq \delta_{\epsilon}
$$

for all $x \in G_{1}$ ?

This question was solved affirmatively by Hyers [2] under the assumption that $G_{2}$ is a Banach space. He proved that if $f$ is a mapping between Banach spaces satisfying $\| f(x+$ $y)-f(x)-f(y) \| \leq \epsilon$ for some fixed $\epsilon \geq 0$, then there exists a unique additive mapping $A$ such that $\|f(x)-A(x)\| \leq \epsilon$. In 1978, Rassias [3] generalized Hyers' result to the unbounded Cauchy difference. Since then, the stability problems of various functional equations have been extensively studied and generalized by a number of authors (see [4-9]).

In particular, Kannappan [10] introduced the following mixed type quadratic-additive functional equation:

$$
f(x+y+z)+f(x)+f(y)+f(z)=f(x+y)+f(y+z)+f(z+x)
$$


and proved that a function on a real vector space is a solution of (1.1) if and only if there exist a symmetric biadditive function $B$ and an additive function $A$ such that $f(x)=$ $B(x, x)+A(x)$. In addition, Jung [11] investigated the Hyers-Ulam stability of (1.1) on restricted domains and applied the result to the study of an interesting asymptotic behavior of the quadratic functions. More generally, Jun and Kim [12] solved the general solutions and proved the stability of the following functional equation, which is a generalization of (1.1):

$$
f\left(\sum_{i=1}^{n} x_{i}\right)+(n-2) \sum_{i=1}^{n} f\left(x_{i}\right)=\sum_{1 \leq i<j \leq n} f\left(x_{i}+x_{j}\right) \quad(n>2) .
$$

Najati and Moghimi [13] introduced another mixed type quadratic-additive functional equation

$$
f(2 x+y)+f(2 x-y)=f(x+y)+f(x-y)+2 f(2 x)-2 f(x)
$$

and investigated the generalized Hyers-Ulam-Rassias stability of this equation in quasiBanach spaces.

In this paper, we introduce the following quadratic-additive functional equation:

$$
\begin{aligned}
& 9 f\left(\frac{x+y+z}{3}\right)+4\left[f\left(\frac{x-y}{2}\right)+f\left(\frac{y-z}{2}\right)+f\left(\frac{z-x}{2}\right)\right] \\
& =3[f(x)+f(y)+f(z)]
\end{aligned}
$$

to establish the general solutions and stability problems of this equation. For real vector spaces $X$ and $Y$, we prove in Section 2 that a mapping $f: X \rightarrow Y$ satisfies (1.2) if and only if there exist a quadratic mapping $Q: X \rightarrow Y$ satisfying

$$
Q(x+y)+Q(x-y)=2 Q(x)+2 Q(y)
$$

and an additive mapping $A: X \rightarrow Y$ satisfying

$$
A(x+y)=A(x)+A(y)
$$

such that

$$
f(x)=Q(x)+A(x)
$$

for all $x \in X$. We refer to [14-24] for the stability results of other mixed type functional equations. In Section 3, we prove the generalized Hyers-Ulam-Rassias stability of (1.2) in Banach spaces.

\section{General solutions of (1.2)}

Throughout this section, $X$ and $Y$ will be real vector spaces. In order to solve the general solutions of (1.2), we need the following two lemmas.

Lemma 2.1 If an even mapping $f: X \rightarrow Y$ satisfies (1.2) for all $x, y \in X$, then $f$ is quadratic. 
Proof Putting $x=y=z=0$ in (1.2), we have $f(0)=0$. Putting $z=-x$ in (1.2) yields

$$
9 f\left(\frac{y}{3}\right)+4\left[f\left(\frac{x-y}{2}\right)+f\left(\frac{x+y}{2}\right)+f(x)\right]=6 f(x)+3 f(y)
$$

for all $x, y \in X$. Replacing $y$ with $x$ in (2.1) gives

$$
9 f\left(\frac{x}{3}\right)=f(x)
$$

for all $x \in X$. Putting $y=0$ in (2.1), we have

$$
4 f\left(\frac{x}{2}\right)=f(x)
$$

for all $x \in X$. Using (2.2) and (2.3), we can rewrite (1.2) as

$$
f(x+y+z)+f(x-y)+f(y-z)+f(z-x)=3[f(x)+f(y)+f(z)]
$$

for all $x, y, z \in X$. Putting $z=0$ in (2.4), we obtain

$$
f(x+y)+f(x-y)=2 f(x)+2 f(y)
$$

for all $x, y \in X$.

Lemma 2.2 If an odd mapping $f: X \rightarrow Y$ satisfies (1.2) for all $x, y \in X$, then $f$ is additive.

Proof Putting $x=y=z=0$ in (1.2), we have $f(0)=0$. Putting $z=-x$ in (1.2) yields

$$
9 f\left(\frac{y}{3}\right)+4\left[f\left(\frac{x-y}{2}\right)+f\left(\frac{x+y}{2}\right)-f(x)\right]=3 f(y)
$$

for all $x, y \in X$. Replacing $y$ by $x$ in (2.5) gives

$$
9 f\left(\frac{x}{3}\right)=3 f(x)
$$

for all $x \in X$. Putting $y=0$ in (2.5), we have

$$
2 f\left(\frac{x}{2}\right)=f(x)
$$

for all $x \in X$. It follows from (2.5), (2.6) and (2.7) that

$$
f\left(\frac{x+y}{2}\right)+f\left(\frac{x-y}{2}\right)=2 f(x)
$$

for all $x, y \in X$. Replacing $x$ with $x+y$ and $y$ with $x-y$ in (2.8), we obtain

$$
f(x+y)=f(x)+f(y)
$$

for all $x, y \in X$. 
Now we are ready to establish the general solutions of (1.2).

Theorem 2.3 A function $f: X \rightarrow Y$ satisfies (1.2) for all $x, y, z \in X$ if and only if there exist a symmetric biadditive mapping $B: X \times X \rightarrow Y$ and an additive mapping $A: X \rightarrow Y$ such that

$$
f(x)=B(x, x)+A(x)
$$

for all $x \in X$.

Proof (Necessity) We decompose $f$ into the even part and the odd part by putting

$$
f_{e}(x)=\frac{1}{2}(f(x)+f(-x)), \quad f_{o}(x)=\frac{1}{2}(f(x)-f(-x))
$$

for all $x \in X$. By Lemmas 2.1 and 2.2 we have the result.

(Sufficiency) This is obvious.

\section{Stability of (1.2)}

In what follows, $X$ and $Y$ will be a real normed linear space and a real Banach space, respectively. For convenience, we define

$$
\begin{aligned}
D f(x, y, z):= & 9 f\left(\frac{x+y+z}{3}\right)+4\left[f\left(\frac{x-y}{2}\right)+f\left(\frac{y-z}{2}\right)+f\left(\frac{z-x}{2}\right)\right] \\
& -3[f(x)+f(y)+f(z)]
\end{aligned}
$$

for all $x, y, z \in X$. Let $\varphi: X \times X \times X \rightarrow[0, \infty)$ be a mapping satisfying one of the conditions $(\mathcal{A}),(\mathcal{B})$ and one of the conditions $(\mathcal{C}),(\mathcal{D})$ :
(A) $\Phi_{1}(x, y, z):=\sum_{k=1}^{\infty} \frac{1}{9^{k}} \varphi\left(3^{k} x, 3^{k} y, 3^{k} z\right)<\infty$,
(B) $\Phi_{2}(x, y, z):=\sum_{k=0}^{\infty} 9^{k} \varphi\left(\frac{x}{3^{k}}, \frac{y}{3^{k}}, \frac{z}{3^{k}}\right)<\infty$,
(C) $\Psi_{1}(x, y, z):=\sum_{k=1}^{\infty} \frac{1}{3^{k}} \varphi\left(3^{k} x, 3^{k} y, 3^{k} z\right)<\infty$,
(D) $\Psi_{2}(x, y, z):=\sum_{k=0}^{\infty} 3^{k} \varphi\left(\frac{x}{3^{k}}, \frac{y}{3^{k}}, \frac{z}{3^{k}}\right)<\infty$,

for all $x, y, z \in X$. We note that the condition $(\mathcal{C})$ implies $(\mathcal{A})$. Similarly, the condition $(\mathcal{B})$ implies $(\mathcal{D})$. One of the conditions $(\mathcal{A}),(\mathcal{B})$ will be needed to derive a quadratic mapping, and one of the conditions $(\mathcal{C}),(\mathcal{D})$ will be required to derive an additive mapping in the following theorem.

Theorem 3.1 Suppose that a mapping $f: X \rightarrow Y$ satisfies

$$
\|D f(x, y, z)\| \leq \varphi(x, y, z)
$$


for all $x, y, z \in X$. Then there exist a unique quadratic mapping $Q: X \rightarrow Y$ satisfying (1.3) and an additive mapping $A: X \rightarrow Y$ satisfying (1.4) such that

$$
\begin{aligned}
& \left\|f(x)-Q(x)-A(x)+\frac{1}{2} f(0)\right\| \\
& \quad \leq \frac{1}{2}\left[\Phi_{i}(x, x,-x)+\Phi_{i}(-x,-x, x)\right]+\frac{1}{6}\left[\Psi_{j}(x, x,-x)+\Psi_{j}(-x,-x, x)\right], \\
& \left\|\frac{f(x)+f(-x)}{2}-Q(x)+\frac{1}{2} f(0)\right\| \leq \frac{1}{2}\left[\Phi_{i}(x, x,-x)+\Phi_{i}(-x,-x, x)\right],
\end{aligned}
$$

and

$$
\left\|\frac{f(x)-f(-x)}{2}-A(x)\right\| \leq \frac{1}{6}\left[\Psi_{j}(x, x,-x)+\Psi_{j}(-x,-x, x)\right]
$$

for all $x \in X$ and for $i=1$ or $2, j=1$ or 2 . The mappings $Q$ and $A$ are given by

$$
\begin{cases}Q(x)=\lim _{n \rightarrow \infty} \frac{1}{2 \cdot 9^{n}}\left[f\left(3^{n} x\right)+f\left(-3^{n} x\right)\right] & \text { if condition }(\mathcal{A}) \text { holds } \\ Q(x)=\lim _{n \rightarrow \infty} \frac{9^{n}}{2}\left[f\left(\frac{x}{3^{n}}\right)+f\left(-\frac{x}{3^{n}}\right)\right], \quad f(0)=0 & \text { if condition }(\mathcal{B}) \text { holds } \\ A(x)=\lim _{n \rightarrow \infty} \frac{1}{2 \cdot 3^{n}}\left[f\left(3^{n} x\right)-f\left(-3^{n} x\right)\right] & \text { if condition }(\mathcal{C}) \text { holds } \\ A(x)=\lim _{n \rightarrow \infty} \frac{3^{n}}{2}\left[f\left(\frac{x}{3^{n}}\right)-f\left(-\frac{x}{3^{n}}\right)\right], \quad f(0)=0 & \text { if condition }(\mathcal{D}) \text { holds }\end{cases}
$$

for all $x \in X$.

Proof We first consider the even part of $f$. Let $f_{e}: X \rightarrow Y$ be a function defined by $f_{e}(x):=$ $\frac{f(x)+f(-x)}{2}$ for all $x \in X$. Then $f_{e}(-x)=f_{e}(x)$ and

$$
\left\|D f_{e}(x, y, z)\right\| \leq \frac{1}{2}[\varphi(x, y, z)+\varphi(-x,-y,-z)]
$$

for all $x, y, z \in X$. Putting $y=x, z=-x$ in (3.2), we have

$$
\left\|9 g\left(\frac{x}{3}\right)-g(x)\right\| \leq \frac{1}{2}[\varphi(x, x,-x)+\varphi(-x,-x, x)]
$$

for all $x \in X$, where $g(x):=f_{e}(x)+\frac{1}{2} f(0)$.

Case 1. Assume that $\varphi$ satisfies the condition $(\mathcal{A})$. Replacing $x$ by $3 x$ in (3.3) and dividing by 9 yield

$$
\left\|g(x)-\frac{g(3 x)}{9}\right\| \leq \frac{1}{2 \cdot 9}[\varphi(3 x, 3 x,-3 x)+\varphi(-3 x,-3 x, 3 x)]
$$

for all $x \in X$. Making use of an induction argument in (3.4) implies

$$
\left\|g(x)-\frac{g\left(3^{n} x\right)}{9^{n}}\right\| \leq \frac{1}{2} \sum_{k=1}^{n} \frac{1}{9^{k}}\left[\varphi\left(3^{k} x, 3^{k} x,-3^{k} x\right)+\varphi\left(-3^{k} x,-3^{k} x, 3^{k} x\right)\right]
$$


for all $n \in \mathbb{N}$ and $x \in X$. From (3.5) we figure out

$$
\begin{aligned}
\left\|\frac{g\left(3^{m} x\right)}{9^{m}}-\frac{g\left(3^{n} x\right)}{9^{n}}\right\| & =\frac{1}{9^{m}}\left\|g\left(3^{m} x\right)-\frac{g\left(3^{n-m} \cdot 3^{m} x\right)}{9^{n-m}}\right\| \\
& \leq \frac{1}{2} \sum_{k=m+1}^{n} \frac{1}{9^{k}}\left[\varphi\left(3^{k} x, 3^{k} x,-3^{k} x\right)+\varphi\left(-3^{k} x,-3^{k} x, 3^{k} x\right)\right]
\end{aligned}
$$

for all $n, m \in \mathbb{N}$ with $n>m$ and $x \in X$. The right-hand side of the inequality above tends to 0 as $m \rightarrow \infty$, the sequence $\left\{g\left(3^{n} x\right) / 9^{n}\right\}$ is a Cauchy sequence for all $x \in X$ and thus converges by the completeness of $Y$. Therefore, we can define a mapping $Q: X \rightarrow Y$ by

$$
Q(x):=\lim _{n \rightarrow \infty} \frac{g\left(3^{n} x\right)}{9^{n}}=\lim _{n \rightarrow \infty} \frac{f\left(3^{n} x\right)+f\left(-3^{n} x\right)}{2 \cdot 9^{n}}
$$

for all $x \in X$. Note that $Q(0)=0, Q(-x)=Q(x)$ for all $x \in X$. It follows from the condition $(\mathcal{A})$ and (3.2) that $Q$ satisfies

$$
9 Q\left(\frac{x+y+z}{3}\right)+4\left[Q\left(\frac{x-y}{2}\right)+Q\left(\frac{y-z}{2}\right)+Q\left(\frac{z-x}{2}\right)\right]=3[Q(x)+Q(y)+Q(z)]
$$

for all $x, y, z \in X$. According to Lemma 2.1, the mapping $Q$ satisfies (1.3). Letting $n \rightarrow \infty$ in (3.5), we have

$$
\|g(x)-Q(x)\| \leq \frac{1}{2}\left[\Phi_{1}(x, x,-x)+\Phi_{1}(-x,-x, x)\right]
$$

for all $x \in X$. Now we are going to prove the uniqueness of $Q$. Assume that $Q^{\prime}$ is another quadratic function satisfying (1.3) and (3.6). Obviously, we have $Q\left(3^{n} x\right)=9^{n} Q(x)$ and $Q^{\prime}\left(3^{n} x\right)=9^{n} Q^{\prime}(x)$ for all $x \in X$. Then we figure out

$$
\begin{aligned}
\left\|Q(x)-Q^{\prime}(x)\right\| & =9^{-n}\left\|Q\left(3^{n} x\right)-Q^{\prime}\left(3^{n} x\right)\right\| \\
& \leq 9^{-n}\left\|Q\left(3^{n} x\right)-g\left(3^{n} x\right)\right\|+9^{-n}\left\|g\left(3^{n} x\right)-Q^{\prime}\left(3^{n} x\right)\right\| \\
& \leq \sum_{k=n+1}^{\infty} \frac{1}{9^{k}}\left[\varphi\left(3^{k} x, 3^{k} x,-3^{k} x\right)+\varphi\left(-3^{k} x,-3^{k} x, 3^{k} x\right)\right]
\end{aligned}
$$

for all $n \in \mathbb{N}$ and $x \in X$. Taking the limit as $n \rightarrow \infty$, we conclude that $Q(x)=Q^{\prime}(x)$ for all $x \in X$.

Case 2. If $\varphi$ satisfies the condition $(\mathcal{B})$ (and hence implies $(\mathcal{D})$ ), the proof is analogous to that of Case 1. By virtue of the condition $(\mathcal{B})$ and (3.1), we have $\varphi(0,0,0)=0$ and $f(0)=0$. An induction argument on (3.3) implies

$$
\left\|9^{n} f_{e}\left(\frac{x}{3^{n}}\right)-f_{e}(x)\right\| \leq \frac{1}{2} \sum_{k=0}^{n-1} 9^{k}\left[\varphi\left(\frac{x}{3^{k}}, \frac{x}{3^{k}},-\frac{x}{3^{k}}\right)+\varphi\left(-\frac{x}{3^{k}},-\frac{x}{3^{k}}, \frac{x}{3^{k}}\right)\right]
$$

for all $n \in \mathbb{N}$ and $x \in X$. Using a similar argument to that of Case 1, we see that the sequence $\left\{9^{n} f_{e}\left(\frac{x}{3^{n}}\right)\right\}$ is a Cauchy sequence for all $x \in X$. Thus we can define a mapping $Q: X \rightarrow Y$ by

$$
Q(x):=\lim _{n \rightarrow \infty} 9^{n} f_{e}\left(\frac{x}{3^{n}}\right)=\lim _{n \rightarrow \infty} \frac{9^{n}}{2}\left[f\left(\frac{x}{3^{n}}\right)+f\left(-\frac{x}{3^{n}}\right)\right]
$$


for all $x \in X$. Note that $Q(0)=0$ and $Q(-x)=Q(x)$ for all $x \in X$. From the condition $(\mathcal{B})$ and (3.2), we see that $Q$ satisfies

$$
9 Q\left(\frac{x+y+z}{3}\right)+4\left[Q\left(\frac{x-y}{2}\right)+Q\left(\frac{y-z}{2}\right)+Q\left(\frac{z-x}{2}\right)\right]=3[Q(x)+Q(y)+Q(z)]
$$

for all $x, y, z \in X$. By Lemma 2.1 the mapping $Q$ satisfies (1.3). Taking the limit as $n \rightarrow \infty$ in (3.7), we obtain

$$
\left\|f_{e}(x)-Q(x)\right\| \leq \frac{1}{2}\left[\Phi_{2}(x, x,-x)+\Phi_{2}(-x,-x, x)\right]
$$

for all $x \in X$. The rest of the proof is similar to that of Case 1 .

Next, we consider the odd part of $f$. Now, let $f_{o}: X \rightarrow Y$ be a function defined by $f_{o}(x):=$ $\frac{1}{2}[f(x)-f(-x)]$ for all $x \in X$. Then $f_{o}(0)=0, f_{o}(-x)=-f_{o}(x)$ and

$$
\left\|D f_{o}(x, y, z)\right\| \leq \frac{1}{2}[\varphi(x, y, z)+\varphi(-x,-y,-z)]
$$

for all $x, y, z \in X$. Putting $y=x, z=-x$ in (3.8) and dividing by 3 yield

$$
\left\|3 f_{o}\left(\frac{x}{3}\right)-f_{o}(x)\right\| \leq \frac{1}{6}[\varphi(x, x,-x)+\varphi(-x,-x, x)]
$$

for all $x \in X$.

Case 3. Assume that $\varphi$ satisfies the condition $(\mathcal{C})$ (and hence implies $(\mathcal{A})$ ). Replacing $x$ by $3 x$ in (3.9) and dividing by 3 , we have

$$
\left\|f_{o}(x)-\frac{f_{o}(3 x)}{3}\right\| \leq \frac{1}{6 \cdot 3}[\varphi(3 x, 3 x,-3 x)+\varphi(-3 x,-3 x, 3 x)]
$$

for all $x \in X$. Making use of an induction argument in (3.10) implies

$$
\left\|f_{o}(x)-\frac{f_{o}\left(3^{n} x\right)}{3^{n}}\right\| \leq \frac{1}{6} \sum_{k=1}^{n} \frac{1}{3^{k}}\left[\varphi\left(3^{k} x, 3^{k} x,-3^{k} x\right)+\varphi\left(-3^{k} x,-3^{k} x, 3^{k} x\right)\right]
$$

for all $n \in \mathbb{N}$ and $x \in X$. From (3.11) we can show that the sequence $\left\{f\left(3^{n} x\right) / 3^{n}\right\}$ is a Cauchy sequence for all $x \in X$. Define a mapping $A: X \rightarrow Y$ by

$$
A(x):=\lim _{n \rightarrow \infty} \frac{f_{o}\left(3^{n} x\right)}{3^{n}}=\lim _{n \rightarrow \infty} \frac{f\left(3^{n} x\right)-f\left(-3^{n} x\right)}{2 \cdot 3^{n}}
$$

for all $x \in X$. By the oddness of $f$, we see that $A(-x)=-A(x)$ for all $x \in X$. Also, from the condition $(\mathcal{C})$ and (3.9), we verify that $A$ satisfies

$$
9 A\left(\frac{x+y+z}{3}\right)+4\left[A\left(\frac{x-y}{2}\right)+A\left(\frac{y-z}{2}\right)+A\left(\frac{z-x}{2}\right)\right]=3[A(x)+A(y)+A(z)]
$$

for all $x, y, z \in X$. According to Lemma 2.2, the mapping $A$ satisfies (1.4). Letting $n \rightarrow \infty$ in (3.11), we have

$$
\left\|f_{o}(x)-A(x)\right\| \leq \frac{1}{6}\left[\Psi_{1}(x, x,-x)+\Psi_{1}(-x,-x, x)\right]
$$


for all $x \in X$. Using a similar argument to that of Case 1, we can easily see the uniqueness of $A$.

Case 4. If $\varphi$ satisfies the condition $(\mathcal{D})$, the proof is analogous to that of Case 3. An induction argument on (3.9) implies

$$
\left\|f_{o}(x)-3^{n} f_{o}\left(\frac{x}{3^{n}}\right)\right\| \leq \sum_{k=0}^{n-1} \frac{3^{k}}{6}\left[\varphi\left(\frac{x}{3^{k}}, \frac{x}{3^{k}},-\frac{x}{3^{k}}\right)+\varphi\left(-\frac{x}{3^{k}},-\frac{x}{3^{k}}, \frac{x}{3^{k}}\right)\right]
$$

for all $n \in \mathbb{N}$ and $x \in X$. It follows from (3.12) that $\left\{3^{n} f\left(3^{-n} x\right)\right\}$ is a Cauchy sequence for all $x \in X$. Thus we can define a mapping $A: X \rightarrow Y$ by

$$
A(x):=\lim _{n \rightarrow \infty} 3^{n} f_{o}\left(\frac{x}{3^{n}}\right)=\lim _{n \rightarrow \infty} \frac{3^{n}}{2}\left[f\left(\frac{x}{3^{n}}\right)-f\left(-\frac{x}{3^{n}}\right)\right]
$$

for all $x \in X$. Note that $A(-x)=-A(x)$ for all $x \in X$. Also, from the condition $(\mathcal{C})$ and (3.8), we verify that $A$ satisfies

$$
9 A\left(\frac{x+y+z}{3}\right)+4\left[A\left(\frac{x-y}{2}\right)+A\left(\frac{y-z}{2}\right)+A\left(\frac{z-x}{2}\right)\right]=3[A(x)+A(y)+A(z)]
$$

for all $x, y, z \in X$. According to Lemma 2.2, the mapping $A$ satisfies (1.4). Taking the limit as $n \rightarrow \infty$ in (3.12), we have

$$
\left\|f_{o}(x)-A(x)\right\| \leq \frac{1}{6}\left[\Psi_{2}(x, x,-x)+\Psi_{2}(-x,-x, x)\right]
$$

for all $x \in X$. Similarly, we can show the uniqueness of $A$.

From the theorem above, we have the following corollary immediately.

Corollary 3.2 Let $p \neq 1, p \neq 2$ and $\epsilon \geq 0$ be real numbers. Suppose that a mapping $f: X \rightarrow$ $Y$ satisfies

$$
\|D f(x, y, z)\| \leq \epsilon\left(\|x\|^{p}+\|y\|^{p}+\|z\|^{p}\right)
$$

for all $x, y, z \in X(x, y, z \in X \backslash\{0\}$ if $p<0)$. Then for each three cases $p<1,1<p<2$ and $p>2$, there exist a unique quadratic mapping $Q: X \rightarrow Y$ satisfying (1.3) and an additive mapping $A: X \rightarrow Y$ satisfying (1.4) such that

$$
\begin{aligned}
& \left\|f(x)-Q(x)-A(x)+\frac{1}{2} f(0)\right\| \leq 3^{p} \epsilon\left(\frac{3}{\left|9-3^{p}\right|}+\frac{1}{\left|3-3^{p}\right|}\right)\|x\|^{p}, \\
& \left\|\frac{f(x)+f(-x)}{2}-Q(x)+\frac{1}{2} f(0)\right\| \leq \frac{3^{p+1} \epsilon}{\left|9-3^{p}\right|}\|x\|^{p},
\end{aligned}
$$

and

$$
\left\|\frac{f(x)-f(-x)}{2}-A(x)\right\| \leq \frac{3^{p} \epsilon}{\left|3-3^{p}\right|}\|x\|^{p}
$$

for all $x \in X(x \in X \backslash\{0\}$ if $p<0)$, where $f(0)=0$ if $p>1$. 
Proof Let $\varphi(x, y, z):=\epsilon\left(\|x\|^{p}+\|y\|^{p}+\|z\|^{p}\right)$ for all $x, y, z \in X$. Then $\varphi(x, x-x)=\varphi(-x,-x, x)=$ $3 \epsilon\|x\|^{p}$ for all $x \in X(x \in X \backslash\{0\}$ if $p<0)$. If $p<2$, the mapping $\varphi$ satisfies $(\mathcal{A})$. Thus, we figure out

$$
\frac{1}{2}\left[\Phi_{1}(x, x,-x)+\Phi_{1}(-x,-x, x)\right]=\sum_{k=1}^{\infty} 3 \epsilon\|x\|^{p} \cdot 3^{(p-2) k}=\epsilon\|x\|^{p} \frac{3^{p+1}}{9-3^{p}}
$$

for all $x \in X(x \in X \backslash\{0\}$ if $p<0)$. If $p>2$, the mapping $\varphi$ satisfies $(\mathcal{B})$. Thus, we have

$$
\frac{1}{2}\left[\Phi_{2}(x, x,-x)+\Phi_{2}(-x,-x, x)\right]=\sum_{k=0}^{\infty} 3 \epsilon\|x\|^{p} \cdot 3^{(2-p) k}=\epsilon\|x\|^{p} \frac{3^{p+1}}{3^{p}-9}
$$

for all $x \in X$. If $p<1$, the mapping $\varphi$ satisfies $(\mathcal{C})$. Thus, we get

$$
\frac{1}{2}\left[\Psi_{1}(x, x,-x)+\Psi_{1}(-x,-x, x)\right]=\sum_{k=1}^{\infty} \epsilon\|x\|^{p} \cdot 3^{(p-1) k}=\epsilon\|x\|^{p} \frac{3^{p}}{3-3^{p}}
$$

for all $x \in X(x \in X \backslash\{0\}$ if $p<0)$. If $p>1$, the mapping $\varphi$ satisfies $(\mathcal{D})$. Thus, we obtain

$$
\frac{1}{2}\left[\Psi_{2}(x, x,-x)+\Psi_{2}(-x,-x, x)\right]=\sum_{k=0}^{\infty} \epsilon\|x\|^{p} \cdot 3^{(1-p) k}=\epsilon\|x\|^{p} \frac{3^{p}}{3^{p}-3}
$$

for all $x \in X$. Therefore, we have

$$
\left\|f(x)-Q(x)-A(x)+\frac{1}{2} f(0)\right\| \leq \begin{cases}3^{p} \epsilon\|x\|^{p}\left(\frac{3}{3^{p}-9}+\frac{1}{3^{p}-3}\right) & \text { if } p>2, \\ 3^{p} \epsilon\|x\|^{p}\left(\frac{3}{9-3^{p}}+\frac{1}{3^{p}-3}\right) & \text { if } 1<p<2 \\ 3^{p} \epsilon\|x\|^{p}\left(\frac{3}{9-3^{p}}+\frac{1}{3-3^{p}}\right) & \text { if } p<1\end{cases}
$$

for all $x \in X(x \in X \backslash\{0\}$ if $p<0)$.

Corollary 3.3 Let $\epsilon \geq 0$ be a real number. Suppose that a mapping $f: X \rightarrow Y$ satisfies

$$
\|D f(x, y, z)\| \leq \epsilon
$$

for all $x, y, z \in X$. Then there exist a unique quadratic mapping $Q: X \rightarrow Y$ satisfying (1.3) and an additive mapping $A: X \rightarrow Y$ satisfying (1.4) such that

$$
\begin{aligned}
& \left\|f(x)-Q(x)-A(x)+\frac{1}{2} f(0)\right\| \leq \frac{7}{24} \epsilon, \\
& \left\|\frac{f(x)+f(-x)}{2}-Q(x)+\frac{1}{2} f(0)\right\| \leq \frac{1}{8} \epsilon,
\end{aligned}
$$

and

$$
\left\|\frac{f(x)-f(-x)}{2}-A(x)\right\| \leq \frac{1}{6} \epsilon
$$

for all $x \in X$. 


\section{Competing interests}

The authors declare that they have no competing interests.

\section{Authors' contributions}

The first author discovered the topic and the main ideas for the proof of the paper and made the actual writing. All authors discussed the paper together. The second and the third authors discovered some helpful ideas for the proof of this paper and checked the proof of the paper. All authors read and approved the final manuscript.

\section{Received: 28 December 2012 Accepted: 19 June 2013 Published: 5 July 2013}

\section{References}

1. Ulam, SM: Problems in Modern Mathematics. Wiley, New York (1964)

2. Hyers, DH: On the stability of the linear functional equation. Proc. Natl. Acad. Sci. USA 27, $222-224$ (1941)

3. Rassias, TM: On the stability of the linear mapping in Banach spaces. Proc. Am. Math. Soc. 72, $297-300$ (1978)

4. Czerwik, S: Functional Equations and Inequalities in Several Variables. World Scientific, River Edge (2002)

5. Găvruta, P: A generalization of the Hyers-Ulam-Rassias stability of approximately additive mappings. J. Math. Anal. Appl. 184, 431-436 (1994)

6. Hyers, DH, Isac, G, Rassias, TM: Stability of Functional Equations in Several Variables. Birkhäuser, Boston (1998)

7. Jung, S-M: Hyers-Ulam-Rassias Stability of Functional Equations in Nonlinear Analysis. Springer Optimization and Its Applications. Springer, New York (2011)

8. Kannappan, P: Functional Equations and Inequalities with Applications. Springer, New York (2009)

9. Sahoo, PK, Kannappan, P: Introduction to Functional Equations. CRC Press, Boca Raton (2011)

10. Kannappan, P: Quadratic functional equation and inner product spaces. Results Math. 27, 368-372 (1995)

11. Jung, S-M: On the Hyers-Ulam stability of the functional equations that have the quadratic property. J. Math. Anal. Appl. 222, 126-137 (1998)

12. Jun, K-W, Kim, H-M: On the stability of an n-dimensional quadratic and additive functional equation. Math. Inequal. Appl. 9, 153-165 (2006)

13. Najati, A, Moghimi, MB: Stability of a functional equation deriving from quadratic and additive functions in quasi-Banach spaces. J. Math. Anal. Appl. 337, 399-415 (2008)

14. Gordji, ME, Ebadian, A, Zolfaghari, S: Stability of a mixed type cubic and quartic functional equation. Abstr. Appl. Anal. 2008, Article ID 801904 (2008)

15. Gordji, ME, Bavand-Savadkouhi, M: Stability of cubic and quartic functional equations in non-Archimedean spaces. Acta Appl. Math. 110, 1321-1329 (2010). doi:10.1007/s10440-009-9512-7

16. Gordji, ME, Kaboli Gharetapeh, S, Rassias, JM, Zolfaghari, S: Solution and stability of a mixed type additive, quadratic and cubic functional equation. Adv. Differ. Equ. 2009, Article ID 826130 (2009)

17. Gordji, ME, Savadkouhi, MB: Stability of mixed type cubic and quartic functional equations in random normed spaces. J. Inequal. Appl. 2009, Article ID 527462 (2009)

18. Gordji, ME, Abbaszadeh, S, Park, C: On the stability of generalized mixed type quadratic and quartic functional equation in quasi-Banach spaces. J. Inequal. Appl. 2009, Article ID 153084 (2009)

19. Gordji, ME, Savadkouhi, MB, Park, C: Quadratic-quartic functional equations in RN-spaces. J. Inequal. Appl. 2009, Article ID 868423 (2009)

20. Gordji, ME: Stability of a functional equation deriving from quartic and additive functions. Bull. Korean Math. Soc. 47 , 491-502 (2010)

21. Gordji, ME, Kaboli Gharetapeh, S, Moslehian, MS, Zolfaghari, S: Stability of a mixed type additive, quadratic, cubic and quartic functional equation. In: Nonlinear Analysis and Variational Problems. Springer Optim. Appl., vol. 35, pp. 65-80. Springer, New York (2010)

22. Lee, Y-S: On the stability of a mixed type functional equation in generalized functions. Adv. Differ. Equ. 2012, 16 (2012)

23. Najati, A, Eskandani, GZ: A fixed point method to the generalized stability of a mixed additive and quadratic functional equation in Banach modules. J. Differ. Equ. Appl. 16, 773-788 (2010)

24. Nakmahachalasint, P: On the generalized Ulam-Gavruta-Rassias stability of mixed-type linear and Euler-Lagrange-Rassias functional equations. Int. J. Math. Math. Sci. 2007, Article ID 63239 (2007)

doi:10.1186/1687-1847-2013-198

Cite this article as: Lee et al.: On the stability of a mixed type quadratic-additive functional equation. Advances in Difference Equations 2013 2013:198. 